\title{
Exploring the relationship between depression and marital satisfaction amongst pregnant women residing in southern Sri Lanka
}

BLE Jayasinha, BN Perera

\section{Abstract \\ Background}

Maternal mental health is considered a global health priority, but despite this, there are still many limitations in addressing issues related to maternal mental health in Sri Lanka. Depression is the most commonly occurring psychiatric disorder among pregnant mothers, and is associated with an increased risk of suicide. Maternal suicides have increased in recent years, with a rate of 12.1 per 100,000 live births in 2010 . There is a pressing need to explore the factors that influence the mental well-being of pregnant mothers.

\section{Aims}

The aim of this study was to explore the relationship between antenatal depression and marital satisfaction.

\section{Methods}

A questionnaire-based survey was conducted among 203 pregnant mothers who presented to 10 maternity clinics in the Galle Municipal area. The Edinburgh Postnatal Depression Scale and the Revised Dyadic Adjustment Scale were used to assess for depression and the level of marital satisfaction, respectively.

\section{Results}

A total of $42.9 \%$ of participants screened positive for antenatal depression, while $5.9 \%$ reported having ideas of self-harm or thoughts of suicide. A statistically significant weak negative correlation was detected, between marital satisfaction and antenatal depression. Lack of support from the husband was identified as significant predictor of depression amongst pregnant mothers.

\section{Conclusions}

The importance of a screening program for antenatal depression and the need for a directed focus on maternal mental health is highlighted in this study. Evidence-based, culturally sensitive mental health interventions would play a significant role in detecting and minimizing maternal mental illnesses, which is likely to help to reduce maternal suicides and minimize associated far-reaching negative impacts.

Key words: Pregnancy, depression, marital satisfaction, Sri Lanka

SL J Psychiatry 2019; 10(1): 4-10

\section{Introduction}

Although pregnancy is often a desired and much anticipated event, it can also be a period of emotional and physical vulnerability. Thus, it is a time when the pregnant woman may need an increased level of physical and psychological support. While the physiological aspect of pregnancy has been prioritized, the associated maternal mental health issues, including maternal suicides, have received comparatively less attention in most health care systems in the world (1).
The Sri Lankan health service has taken many steps in recent years, to improve the safety of pregnancy and childbirth, reducing the maternal mortality rates to 30 per 100000 in 2015, which is on par with most developed countries (2). In contrast, the rates of maternal suicide have shown an upward trend in the years 2002-2010, reaching a rate of 12.1 per 100,000 live births in 2010 (3).

Suicide is a leading cause of death among pregnant mothers around the world (4). Oates reported that $10 \%$ of maternal mortality was due to suicide and that $86 \%$ of 
maternal suicides were associated with a psychiatric diagnosis in the UK (5). Worldwide, $10 \%$ of pregnant mothers, and in developing nations $15.6 \%$ of pregnant mothers, are estimated to have psychiatric disorders (6). Studies indicate that psychological disorders, mainly maternal depression and maternal psychosis, strongly influence morbidity and suicide mortality during pregnancy $(5,7)$. Antenatal depression (AD) has far reaching detrimental effects; $\mathrm{AD}$ results in substantial reduction in the quality of life and the functioning capacity of the mother (8). The mother's psychological distress and low mood may also adversely affect the growing fetus, increasing the probability of poor neonatal outcomes and miscarriages (9).

While ideally, the precise identification of the biopsychosocial causes for AD is important in screening and designing preventive programs, the picture is complicated by the multifactorial etiology of depression in the antenatal period.

A meta-analysis focusing on etiology has reported that empathetic and supportive husbands are helpful in reducing the distress, whereas marital dissatisfaction makes the pregnant mothers more vulnerable to depression (1). Research evidence indicates a statistically significant difference in marital satisfaction between depressed and non-depressed expecting mothers (10). Thus, partner support and marital satisfaction seem to be important determinants of AD.

Given these international findings, the aim of the current study is to investigate the relationship between marital satis-faction and depression in pregnant mothers, in Sri Lanka. This study also explores the association between different demographic factors and the occurrence of AD. These findings are likely to indicate what measures should be taken to improve maternal mental health, by researchers, policymakers and healthcare professionals.

\section{Methods}

Data collection was conducted over a one-month period using convenience sampling, based on the availability of the researcher during maternity clinics. All participants were fully informed about the study via one to one information sessions and an information sheet. Those who gave written informed consent were included in the study. Pregnant mothers in any trimester, were considered eligible for the study. Women who could not understand either Sinhalese or English, were excluded. Only those who gave written informed consent were included in the study.

A questionnaire-based survey was carried out using the Edinburgh Postnatal Depression Scale (EPDS), the Revised Dyadic Adjustment Scale (RDAS) and a questionnaire designed for the study, to collect socio- demographic details. The EPDS is a 10-item scale, where a score higher than 9 indicates a high risk of having depression. The RDAS is a 14-item scale with three subsections. The first subscale, marital consensus, explores the degree of agreement between marital partners and a score lower than 22 depicts poor marital agreement. The second subscale, marital satisfaction, examines the degree of fulfilment in married life and a score lower than 14 depicts poor marital satisfaction. The third subscale, marital cohesion, tests the degree of unity and organization, and a score lower than 11 indicates poor cohesion in activities. The combination of all three subscales, i.e., the dyadic adjustment, tests the degree of relationship adjustment and a total score lower than 48 indicates poor dyadic adjustment.

The questionnaires were self-administered, but if the participant was unable to read and understand either Sinhalese and English while being fluent in the spoken language, the researcher filled out the questionnaires based on the participant's verbal responses.

The English versions of the EPDS and RDAS were subjected to translation and back translation, to ensure accurate translation of the original content. Further, the content of the RDAS were superficially reviewed for the appropriateness to the study purpose by the author (BNP) of this research project.

All completed questionnaires were kept in a file, to ensure anonymity and to minimize researcher bias. Ethical clearance for the study was obtained from the Ethics Committee of the University of West London and permission was also obtained from the Regional Director of Health Services, Galle, to proceed with data collection at the Municipal-Medical Officer of Health clinics.

Data were analysed using SPSS version 24. Both descriptive and inferential statistical analyses were conducted. Spearman's correlational analyses were conducted to assess the strength and significance of the associations between variables. The variations of depression level and marital satisfaction were tested in relation to different demographic factors using a series of Kruskal-Wallis H tests and Mann-Whitney U tests. A multiple regression analysis was conducted to explore the effect of confounding variables in determining the relationship between $\mathrm{AD}$ and marital satisfaction.

\section{Results}

Participants included 203 pregnant mothers from 10 different clinics, in 7 different Public Health Midwife areas. Participants included 46, 87 and 60 mothers in their first, second and third trimesters respectively. Participants were of Sinhalese, Tamil and Muslim ethnicity. The age range of this cohort of pregnant mothers was $18-42$ years, with a mean age of 29.5 years $(\mathrm{SD}=4.86)$. 
The mean score, standard deviation, maximum score and the minimum score for the levels of depression and each RDAS variable (marital satisfaction, dyadic adjustment, marital cohesion and marital consensus) are given in the Table 1 . Of the total sample, $5.9 \%$ of the participants $(n=12)$ reported having either ideas of harming themselves or suicidal thoughts or behaviours.

The Shapiro-Wilk test of normality revealed that all test variables were not normally distributed and therefore, to explore the associations, Spearman's correlations were carried out. Partial correlations were carried out between the two main variables, depression and marital satisfaction, by treating the duration of marriage as a confounding variable and by treating both age and the duration of marriage as confounding variables (Table 2). As the $r$ value changed when the duration of marriage was controlled for, it was a confounding variable in the association between $\mathrm{AD}$ and marital satisfaction. However, when both age and the duration of marriage were controlled, no change in the correlation coefficient was observed.

To explore the variation of mean depression scores and mean RDAS scores between groups in relation to different demographic variables, a series of MannWhitney U tests and Kruskal-Wallis H tests were carried out (Table 3). Mann-Whitney U tests carried out to check the significance of these differences revealed that none were statistically significant.

The Kruskal-Wallis H test revealed that depression levels between different income categories differed significantly $\left(\chi^{2}=12.04, p=0.007\right)$, with the highest mean rank being reported by the highest income category. There were no significant differences in the occurrence of depression and RDAS variables among different categories based on the level of education of the participant, or her husband, or the trimester of the pregnancy.

\section{Table 1. Levels of depression and scores for the RDAS scale $(\mathrm{N}=203)$}

\begin{tabular}{|l|c|c|c|c|c|}
\hline $\begin{array}{l}\text { Descriptive } \\
\text { Measure }\end{array}$ & Depression & $\begin{array}{c}\text { Marital } \\
\text { Satisfaction }\end{array}$ & $\begin{array}{c}\text { Marital } \\
\text { Cohesion }\end{array}$ & $\begin{array}{c}\text { Marital } \\
\text { Consensus }\end{array}$ & $\begin{array}{c}\text { Dyadic } \\
\text { Adjustment }\end{array}$ \\
\hline$M$ & 8.73 & 17.93 & 16.51 & 26.40 & 60.84 \\
\hline$S D$ & 4.34 & 2.48 & 2.54 & 3.37 & 6.56 \\
\hline Minimum Score & 0.00 & 4.00 & 4.00 & 13.00 & 35.00 \\
\hline Maximum Score & 23.00 & 20.00 & 19.00 & 30.00 & 69.00 \\
\hline Above cut-off & $n=87(42.9 \%)$ & $n=194(95.6 \%)$ & $n=195(96.1 \%)$ & $n=185(91.1 \%)$ & $n=191(94.1 \%)$ \\
\hline Below cut-off & $n=116(57.1 \%)$ & $n=9(4.43 \%)$ & $n=8(3.94 \%)$ & $n=18(8.87 \%)$ & $n=12(5.91 \%)$ \\
\hline
\end{tabular}

Note. $M=$ Mean, $S D=$ Standard deviation, $n=$ Number of participants

\begin{tabular}{|l|c|c|}
\hline \multicolumn{1}{|c|}{ Table 2. Coefficients and significances of correlational analyses between depression and } \\
RDAS variables ( $N=203)$ & Correlation coefficient \\
\hline Variables under analysis & Control Variable & $r_{s}=-0.44^{* *}$ \\
\hline Depression and marital satisfaction & - & $r_{s}=-0.39^{* *}$ \\
\hline Depression and dyadic adjustment & - & $r_{s}=-0.27^{* *}$ \\
\hline Depression and marital cohesion & - & $r_{s}=-0.20^{* *}$ \\
\hline Depression and marital satisfaction & Duration of marriage & $r_{s}=-0.45^{* * *}$ \\
\hline Depression and marital satisfaction & Duration of marriage and age & $r_{s}=-0.44^{* * *}$ \\
\hline
\end{tabular}

Note. ${ }^{* *}=p<0.01,{ }^{* * *}=p<0.001$ 
The Kruskal-Wallis $\mathrm{H}$ tests revealed significant differences in levels of depression $\left(\chi^{2}=36.18, p<0.000\right)$, marital satisfaction $\left(\chi^{2}=35.03, p<0.000\right)$, marital cohesion $\left(\chi^{2}=33.57, p<0.000\right)$, marital consensus $\left(\chi^{2}=21.44\right.$, $p<0.000)$ and dyadic adjustment $\left(\chi^{2}=46.10, p<0.000\right)$ between different groups based on the levels of support from the husband.
Additional predictors, age and the duration of marriage were explored in a hierarchical multiple regression analysis. All three models were significant. Marital satisfaction predicted $19.8 \%$ of the variance in depression $\left(\mathrm{R}^{2}\right.$ change $\left.=20.2 \%\right)$. Age $\left(\mathrm{R}^{2}\right.$ change $\left.=0.1 \%\right)$ and duration of marriage $\left(\mathrm{R}^{2}\right.$ change $\left.=0.4 \%\right)$ each predicted $19.5 \%$ of the variance in depression (Table 4).

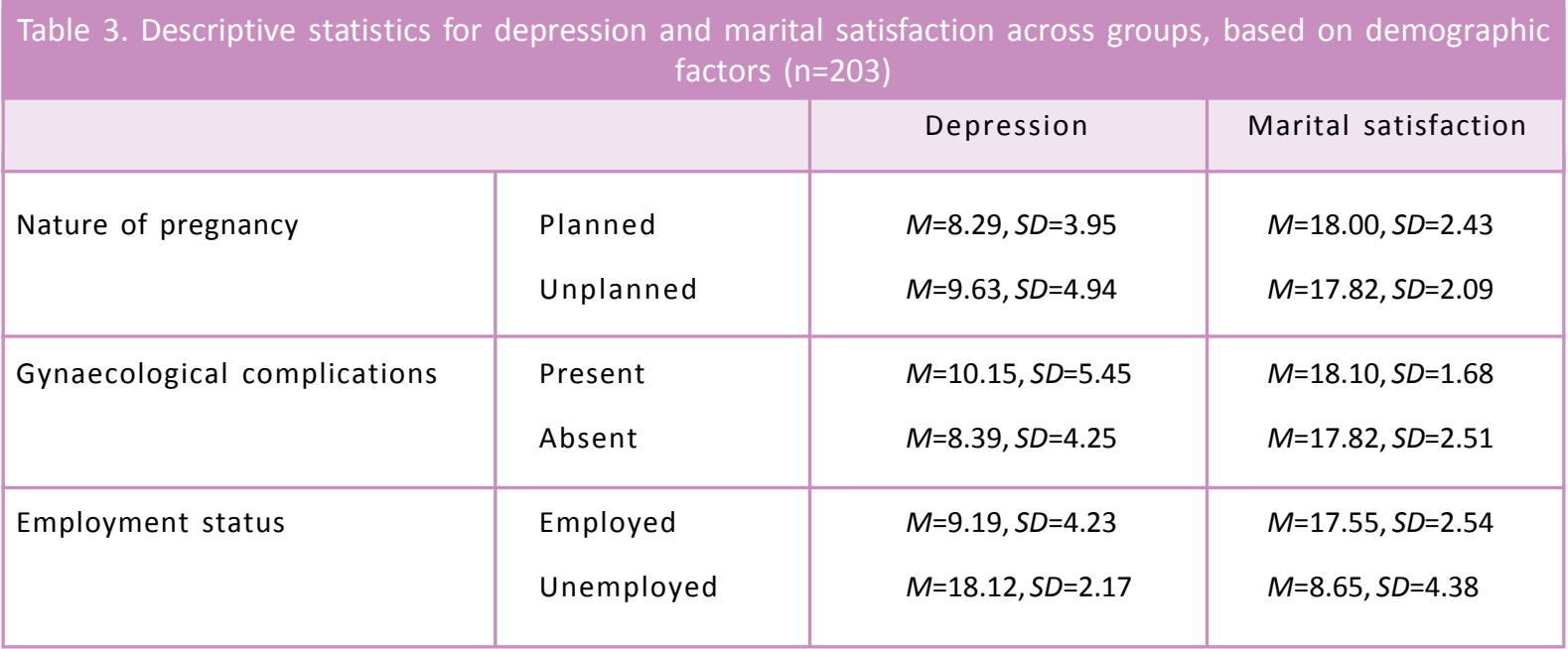

Note. $M-$ mean, $S D-$ standard deviation

Figure 1. Scatter plot of marital satisfaction versus depression.

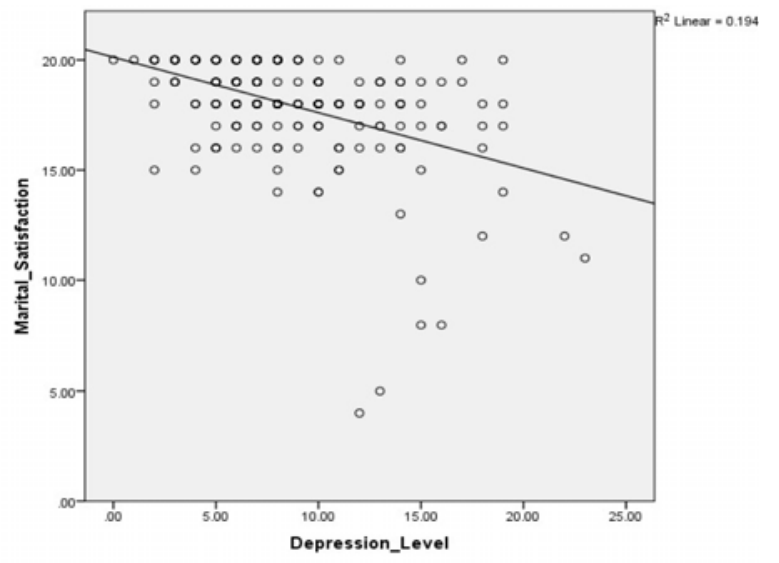

Higher scores indicate higher levels of marital satisfaction and depression, respectively.

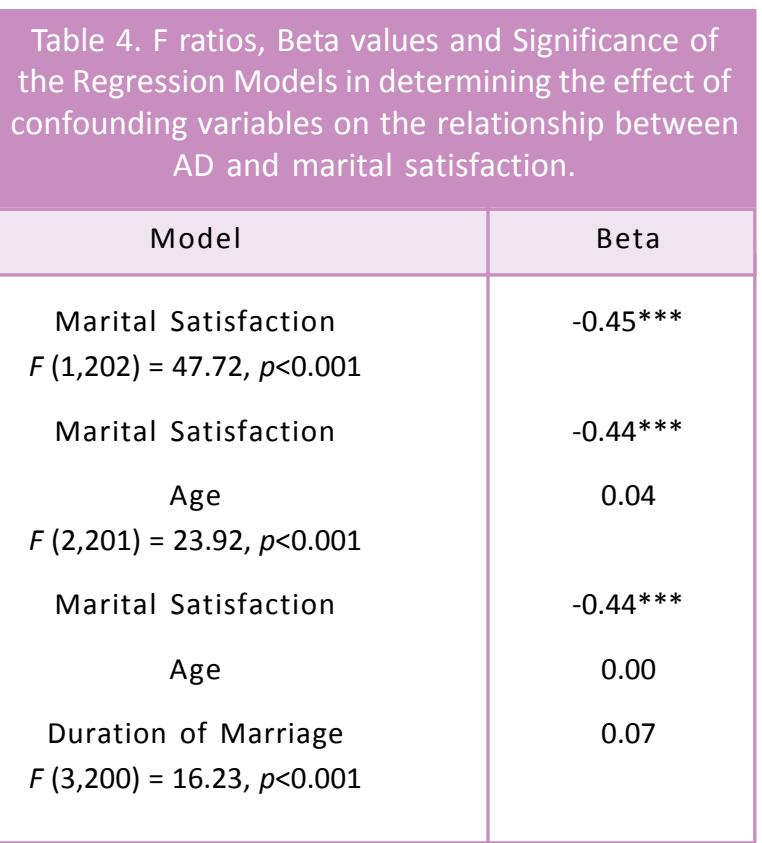

Note. $F=F$ ratio, $p=$ Statistical significance, $* * *=p<0.001$ 


\section{Discussion}

A significant proportion (42.9\%) of the current study sample reported depression scores that require support from a mental health professional. However past studies conducted in some other localities in the country revealed relatively lower rates of $\mathrm{AD}$. For instance, the occurrence of $\mathrm{AD}$ was reported to be $16 \%$ in Anuradhapura, 22.8\% in Galigamuwa, and $25.6 \%$ in the Puttalam district (11-13). Differences in study methodology and techniques of assessing for depression may have contributed to this difference. Socio-demographic differences in different parts of the country may have also played a role. These findings overall indicate the need for increased awareness of depression among pregnant mothers, with greater emphasis on screening, identification and support of such mothers.

The literature supports the predominance of depression and other mental health issues among mothers from low socio-economic status $(14,15)$. Contrastingly, in this study, those from a higher economic class had the highest mean depression scores, even though participants from the lowest economic class (monthly income below 9999/rupees) also reported a mean depression score above the average. The lack of social support and economic stressors among those in the lower social class, and perhaps a sense of social disconnect amongst those from a higher social class, may have contributed to these differences, and these factors should be explored further (16).

Employment may increase the risk of depression due to the strain of managing multiple roles, such as the responsibility of managing occupation and household responsibilities, aggravated by increased fatigue and physiological changes. Nevertheless, economic safety and thus, access to high-quality health care services can benefit the employed women whereas unemployment results in economic insecurity and even poverty. Poverty has been identified as one of the crucial determinants of depression mediated through blockage of access to highquality health services $(17,18)$. Contrastingly, in this study, a higher percentage of employed mothers scored positive for depression, compared to unemployed mothers.

Unplanned pregnancies may increase the risk of AD due to the distress associated with the unexpectedness of the pregnancy. Unplanned pregnancies have been found to be common amongst low income and poorly educated groups (19). Consistent with previous studies, in this study too, a higher percentage of participants with unplanned pregnancies scored positive for depression, compared to participants with planned pregnancies (20-21).

Evidence regarding the variation of AD across the three trimesters of pregnancy is mixed. Some studies report that depression is more frequent and severe during the first trimester, with a decreasing trend towards the second trimester and again an increasing trend with the approach of delivery (22). An increasing trend of AD from the first to the second trimester has also been described (23). In the current study, depression showed a decreasing trend across the three trimesters. Being new to the experience in the case of first-time mothers, and unpreparedness in the case of unplanned pregnancies may have aggravated the risk of $\mathrm{AD}$ in the first trimester.

A Pakistani study reported that women were more satisfied in the marriage of their choice compared to women in arranged marriages (25). High marital quality in 'love marriages' were ascribed to better mutual understanding (25). The relationship between the type of marriage and marital satisfaction, however, is greatly influenced by the culture (26). Furthermore, AD and marital satisfaction may influence each other resulting in a bidirectional relationship. Therefore, the cause-effect relationship between them is unpredictable. In the current study cohort, there was a weak negative association between marital satisfaction and the occurrence of AD (Figure 1). Even though weakly negative, this significant correlation suggests that improving marital satisfaction can be considered in mental health promotion campaigns and interventions.

A large-scale study in Norway conducted amongst 49,425 pregnant mothers explored 37 risk factors in relation to maternal distress and found marital dissatisfaction to be the strongest predictor, revealing the importance of marital satisfaction in maternal mental health (1). This is further supported by findings from a multiple regression analysis from Iran, which found that marital satisfaction and social support together predicted $15.3 \%$ of the variance in depression (27). In the current study, marital satisfaction alone predicted $19.8 \%$ of the variance in the depression score, indicating that marital satisfaction is an important factor for maternal depression in Sri Lanka.

Despite the responsibility men have in women's health, a Sri Lankan study focusing on paternal knowledge reported that fathers have very poor knowledge about pregnancy and maternal morbidity (28). Thus, lack of support from the husband and lack of understanding may be important considerations in maternal mental health.

In the Sri Lankan society, with it's range of cultural taboos, having a psychological problem or illness may be considered a disgrace or a shame. Consequently, people do not seek treatment and are undiagnosed in many instances.

As maternal mental health is relatively less explored in the Sri Lankan context, in depth qualitative studies and national-level studies would be vital to get a clear picture of factors related to depression and maternal suicide. 
Factors such as substance abuse and intimate partner violence are other factors to consider when determining the role of marital dissatisfaction in $\mathrm{AD}$.

\section{Limitations}

Although the sample is a good representation of the Galle Municipal Council area, the results cannot be generalized beyond this locality, to the whole of Sri Lanka. Further, use of convenient sampling techniques might have influenced the results, making it less generalizable.

The fact that this study relied on the self-reported data may have adversely impacted the accuracy of the findings. Response bias is highly probable especially due to the stigmatized nature of the topic. Sensitive yet important gender-based risk factors like intimate partner violence and abuse in childhood were not explored in this study to minimize the risk of evoking distress in the participants, which is a limitation in the findings.

Even though a locally validated Sinhalese version of EPDS is available, this was not used; however, the researcher took steps to minimize errors and biases through translation-back translation procedure.

\section{Conclusions}

A relatively large proportion of the participants in this study screened positive for $\mathrm{AD}$, and a smaller group reported having ideas of self-harm or suicidal thoughts. The level of AD and marital satisfaction showed a significant weakly negative association. Thus, enhancing marital satisfaction may reduce the risk of depression in pregnant mothers. Risk factors and protective factors seem to show greater diversity amongst pregnant mothers. Thus conducting further research and further developing systematic mental health care programs for pregnant mothers is an ongoing need in the country. Furthermore, paternal knowledge about pregnancy and the antenatal period has been shown to be poor in Sri Lanka. Therefore, educating and engaging men is also a key part of improving partner support for pregnant women. Although challenging, exploring culturally appropriate and innovative ways to educate and engage both men and women about planning of a pregnancy, support during pregnancy, and increased awareness and detection of mental health problems in pregnancy, is likely to help reduce the burden of AD in the country.

\section{Acknowledgements}

The authors gratefully acknowledge the support given by Dr. Mojgan Shadbash for this research project. The authors also express sincere thanks to the Galle Regional Director, Health Services, the MOH-Galle Municipal Council staff, all doctors and midwives, pregnant women and participants, and all others who contributed to this study.

\section{Statement of contribution}

The development of the project, data collection, analysis and writing were done by the first author, LJ. Mentoring, supervision and validation of the questionnaires were done by NP. Both authors have seen and approved the final version of the article.

\section{Conflicts of interest}

None declared

BLE Jayasinha, BN Perera, University of West London Corresponding author: BLE Jayasinha

Email: lakshanierangika@gmail.com

http://orcid.org/0000-0002-1175-1710

\section{References}

1. Røsand GM, Slinning K, Eberhard-Gran M, Røysamb E, Tambs K. Partner relationship satisfaction and maternal emotional distress in early pregnancy. BMC public health 2011; 11(1): 161.

2. Isuru LLA, Gunathillaka KDK, Kathriarachchi ST. Reducing maternal suicide in Sri Lanka: closing the gap. SLJ Psychiatry 2016; 7(1).

3. Jayaratne K. Maternal suicides in Sri Lanka: Lessons learnt from review of maternal deaths over 9 years (2002-2010). In: Suicide in Sri Lanka: Past, present and future transformations. Colombo: World Health Organization; 2013.

4. The global burden of disease: 2004 update [Internet]. World Health Organization. World Health Organization; 2014. Available from: https://www.who.int/healthinfo/ global_burden_disease/2004_report_update/en/

5. Oates M. Perinatal psychiatric disorders: a leading cause of maternal morbidity and mortality. Br Med Bull 2003; 67(1): 219-29.

6. Rahman A, Fisher J, Bower P, et al. Interventions for common perinatal mental disorders in women in low- and middle-income countries: a systematic review and metaanalysis. Bull World Health Organ. 2013; 91(8).

7. Rishard MR, Ranaweera AK, Senanayake HM. Can we reduce maternal suicides? SLOG 2012; 34(3): 123.

8. World Health Organization. Maternal mental health and child health and development in resource-constrained settings: Report of a UNFPA. Geneva: World Health Organization; 2007. 
9. Glover V. Maternal stress or anxiety in pregnancy and emotional development of the child. The Br J Psychiatry 1997; 171(2): 105-6.

10. Kiani F, Khadivzadeh T, Sargolzaee MR, Behnam H. Relationship between marital satisfaction during pregnancy and postpartum depression (PPD). Iran J Obstetrics, Gynecology and Infertility 2010; 13(5): 37-44.

11. Agampodi SB, Agampodi TC. Antenatal depression in Anuradhapura, Sri Lanka and the factor structure of the Sinhalese version of Edinburgh postpartum depression scale among pregnant women. PloS one 2013; 8(7): e69708.

12. Jayakody JMSN, Hemachandra NM. Prevalence and psychosocial correlates of antenatal depression among pregnant women in $\mathrm{MOH}$ area, Galigamuwa. In: International conference on global public health; 2014.

13. Rowel D, Jayawardena P, Fernando N. Validation of the Sinhala translation of Edinburgh Postnatal Depression Scale. Ceylon Medical Journal 2008; 53: 10-3.

14. Bennett HA, Einarson A, Taddio A, Koren G, Einarson TR. Prevalence of depression during pregnancy: systematic review. Obstet Gynecol 2004; 103(4): 698-709.

15. Holzman C, Eyster J, Tiedje LB, Roman LA, Seagull E, Rahbar MH. A life course perspective on depressive symptoms in mid-pregnancy. Matern Child Health J 2006; 10(2): 127.

16. Sato Y, Zenou Y. How urbanization affect employment and social interactions. Eur Economic Rev 2015; 75: 131-55.

17. Park CM, Seo HJ, Jung YE, et al. Factors associated with antenatal depression in pregnant Korean females: the effect of bipolarity on depressive symptoms. Neuropsychiatr Dis Treat 2014; 10: 1017.

18. Rwakarema M, Premji SS, Nyanza EC, Riziki P, PalaciosDerflingher L. Antenatal depression is associated with pregnancy-related anxiety, partner relations, and wealth in women in Northern Tanzania: a cross-sectional study. BMC Women's health 2015; 15(1): 68.

19. Christensen AL, Stuart EA, Perry DF, Le HN. Unintended pregnancy and perinatal depression trajectories in lowincome, high-risk Hispanic immigrants. Prev Sci 201; 12(3): 289-99.

20. Lau Y, Keung DW. Correlates of depressive symptomatology during the second trimester of pregnancy among Hong Kong Chinese. Soc Sci Med 2007; 64(9): 1802-11.

21. Nakku JN, Nakasi G, Mirembe F. Postpartum major depression at six weeks in primary health care: prevalence and associated factors. Afr Health Sci 2006; 6(4): 207-14.

22. Öhman SG, Grunewald C, Waldenström U. Women’s worries during pregnancy: testing the Cambridge Worry Scale on 200 Swedish women. Scand J Caring Sci 2003;17(2): 148-52.

23. Hoffman S, Hatch MC. Depressive symptomatology during pregnancy: evidence for an association with decreased fetal growth in pregnancies of lower social class women. Health Psychol 2000; 19(6): 535.

24. Dhillon N, MacArthur C. Antenatal depression and male gender preference in Asian women in the UK. Midwifery 2010; 26(3): 286-93.

25. Arif N, Fatima I. Marital Satisfaction in different types of marriage. Pak J Soc Clin Psychol 2015; 13(1): 36.

26. Cohen TF, Strong B, DeVault C. The marriage and family experience: Intimate relationships in a changing society. Belmont, CA: Wadsworth; 2013.

27. Noury R, Karimi N, Mohammadi M. Relationship between prenatal depression with social support and marital satisfaction. SJRM 2017; 1 (4): 153-7.

28. Weerakkody A, Weerasinghe GM, Weerasinghe MP, Weerasekara GL, Agampodi SB. Expectant fathers' knowledge of maternal morbidity: A Sri Lankan experience. F1000 Res 2013; 2: 119. 